\title{
ASEISMIC DESIGN OF TURBINE HOUSES OF NUCLEAR POWER PLANTS
}

\author{
(ASEISMISCHE AUSLEGUNG DER \\ MASCHINENHÄUSER VON KERNKRAFTWERKEN)
}

\author{
R. DANISCH and M. LABES \\ Kraftwerk Union AG, Kraftwerktechnik, D-852 Erlangen, Germany
}

\section{SUMMARY}

The turbine house does not belong to the safety-related parts of equipment of a nuclear power plant. A special protection against earthquakes is not demanded by the authorities as long as it is proven that safety-related parts of equipment will not be restricted in their function by a collaps of the turbine house. The degree of an aseismic design is largely up to the customer, who has to weigh the risk of costs and availability against the additional costs, that are necessary. for the earthquake calculation and for constructive hardening.

In comparison to the high-tuned turbine foundations as they are in use in the USA today, low-tuned turbine foundations as a result of helical-spring-support, which are constructed by the KWU exclusively, pose special problems with the aseismic design. This will be discussed in particular in the presented report.

The spring-supported mass constitutes about a quarter of the building-mass. For mechanical reasons the spring elements are chosen in such a way, that the turbine foundation has a natural frequency of $-3 \mathrm{~Hz}$. Thus it remains within the same frequency range as the turbine house and within that very range which is particularly amplificated by an earthquake. It is therefore likely that resonance effects as well as oscillation annulment effects may occur.

The standardized calculation methods for conventional buildings without safety function such as DIN 4149 (Germany) or SIA 162 (Switzerland) do not cover the oscillation conduct of such a complicate structure. One receives informations about possible relative displacements between the building and the turbine foundation (hammering-effect) and about the stresses on the turbine and other components only by dynamic calculation methods such as the time-history or the response-spectrum method.

In dependence of the assumed earthquake (operating basis earthquake) the following constructive hardening measures will be necessary:

small earthquakes $(<0.05 \mathrm{~g})$

- reinforcement of the building structure, the concept of the building structure may be kept unchanged.

medium earthquakes

- reinforcement of the building structure, slight alterations of the building concept;

- installation of damping elements at the spring assemblies;

- reinforcement of the turbine foundation without changing the concept.

strong earthquakes $(>0,15 \mathrm{~g})$

- essential altcration of the building concept such as light steel construction or renunciation of the machine hall, additional shear-walls, deeper foundation level of the building;

- installation of damping elements at the spring assemblies;

- reinforcement of turbine bearings;

- modified design of the turbine foundation.

The acceleration values given above are only a rough evidence, since the stresses are largely dependent on particular soil conditions and response-spectra of the site. 


\section{Einle1tung}

Das Maschinenhaus gehört zu den sicherheitstechnisch nicht relevanten Anlagentellen elnes Kernkraftwerkes. Der Grad der erdbebensicheren Auslegung liegt daher weltgehend 1m Ermessen des Betreibers, der das Kostenund Verfugbarke1tsrisıko abzuwägen hat gegenuber dem Mehraufwand, der für die Erdbebenberechnung und konstrukt1ven Ertiuchtigungen erforderlich wird.

In diesem Berlcht wird zunächst die allgemeine Problemat1k der Erdbebenauslegung von Maschinenhäusern behandelt. AnschlleBend werden die Ergebnisse elner Parameteruntersuchung erläutert.

\section{Beschrefloung eines Maschinenhauses}

\subsection{Gebäude}

Das Maschinenhaus 1st üblicherweise eln kastenförmiger, auf elner durchgehenden Fundamentplatte gegrindeter Baukörper, der durch Stahlbetonscheiben und durch Rahmen ausgeste1ft 1st. Die Innenkonstruktion besteht aus Rahmen und Deckenplatten, d1e große Öffnungen zur Montage und zur Aufnahme der Komponenten aufwe1sen. Typische Gebäudeschnitte sind In den Bildern 1 und 2 dargestellt.

\subsection{Turbosatz}

Der Turbosatz besteht aus elnem Hochdruckte11, mehreren Niederdrucktellen und den zugehörigen Kondensatoren sowie dem Generator und der Erregermaschine. Die Drehzahl beträgt in Europa $25 \mathrm{~s}^{-1}$ oder $50 \mathrm{~s}^{-1}$, das Arbeltsmedium ist Sattdampf.

Von einigen Ausnahmen abgesehen, ist es in den USA üblich, hoch abgestimmte Turbinenfundamente zu beuen. Die unterste Elgenfrequenz dieser Fundamente liegt uber der Drehzahl der Turbine und somit in elnem Frequenzbere1ch, der durch Erdbebenerschutterungen nicht angeregt wird, Für die aselsmlsche Auslegung kann das Fundament als starr angesehen werden, Resonanzerscheinungen treten nicht auf. Es ergeben sich keine spezifischen Probleme, die anders wären, als bel üblichen Hochbauten.

Im Gegensatz zu dieser Bauweise werden in Dẹtschland seit ca. 25 Jahren nahezu nur tief abgestimmte Turbinenfundamente gebaut, deren unterste Eigenfrequenz unterhalb der Turbinendrehzehl liegt. Erreicht wurde diese Abst1mmung durch Trägerrostkonstruktionen auf schlanken Stutzen, sogenannten T1schfundamenten. M1t dem Bau von großen Sattdampfmaschinen fur Kernkraftwerke mit einer Drehzahl von $25 \mathrm{~s}^{-1}$ wurde es schwierlg, diese tiefe Abstimmung wegen der dafur erforderlichen Schlankhe1t mit Stahlbetonstutzen zu erzielen. Aus diesem Grunde entschloß man 
sich, die Funktion der Stahlbetonstützen durch Federelemente zu ersetzen, zumal diese Art der Unterstützung für kleinere Maschinen wie Notstromdiesel, Kohlemihlen oder Pumpen seit langem bekannt 1st und sich bewahrt hat.

Fur die Anordnung der Federelemente glbt es verschiedene Möglichkelten, wie zum Beisplel in Reihen auf Unterstitzungslängsbalken bel Turbinen fur Siedewasserreaktoranlagen, oder konzentriert auf stützenköpfen be1 Turblnen fidr Druckwasserreaktoranlagen.

Die Kondensatoren ruhen ebenfalls auf Federn und sind, wie in Europa ubl1ch, fest m1t dem Turbinengehäuse verbunden. Turbine, Generator und Kondensator bilden mit dem Turbosatzfundament elne federnd gelagerte Masse von mehreren zehntausend Meganewton, die in etwa einem Viertel der Gebäudemasse entspricht.

Die Stelfigkelten der Federelemente werden aus maschinentechnischen Gründen so gewählt, daß das Turbinenfundament elne unterste Eigenfrequenz von ca. 2 bis $3 \mathrm{~s}^{-1}$ in vertikaler und horlzontaler Richtung hat. Es liegt damit $1 \mathrm{~m}$ gleichen Frequenzbereich wie das Maschinengebäude, elnem Berelch, der durch Erdbeben vornehmllch angeregt wird. Es kann daher sowohl zu Resonanzeffekten als auch aufgrund der groBen abgefederten Massen zu Schwingungst1lgungseffekten kommen.

Weltere Elnzelheiten der Bauweise von auf Federn gelagerten Turbinenfundamenten sind in [1] beschrieben.

\section{Auslegung gegen Erdbeben}

\subsection{Quasistat1sche Methoden}

An Standorten mit geringem seismischen R1s1ko, wie z.B. Im norddeutschen Kistengebiet, 1st es uiblich, nur slcherheitstechnisch w1chtigo Anlagentelle gegen Eräbeben auszulegen und auf elnen Nachweis für andere Bauteile zu verzichten.

Maschinenhäuser in erdbebengefährdeten Gebieten wurden bisher nach einfachen, sogenannten quasistatischen Methoden bemessen. Belspiele dafur sind die Maschinenhäuser flir die Kernkraftwerke B1blis A und B, die nach der deutschen Erdbebennorm DIN 4149 ausgelegt wurden. Nach dieser Norm werden je nach Erdbebenzone und Baugrund horizontale Ersatzlasten als konstanter Antell der Gebäude- und Komponentenlasten in der statischen Berechnung berúcks1chtigt. Vertıkale Zusatzlasten werden vernachlässigt, we1l sie erfahrungsgemäß bel Erdbeben mitteleuropäischer stärke zu kelnen Schäden führen. Dieser Ansatz bedeutet elne liber die Gebäudehöhe konstant 
angenommene horizontal wirkende Beschleunigungsverteilung, ohne Berickslchtigung des Schwingungsverhalten des betrachteten Systems, was sowohl zu einer Uber- als auch Unterschätzung der Erdbebenlasten fihren kann. Andere Normen, wie der französische Erdbebencode [2] oder die Richtlinien für das Bauen in Erdbebengebieten des Landes Baden-Wirttemburg (BRD) erfassen das dynamische Verhalten zumindest iberschlägllch, indem bei der Berechnung der Beschleunigungsverteilung eln Faktor in Abhängigkeit der Eigenschwingzelt beruicksichtigt wird. Mit diesen Näherungsmethoden erhält man fur ibliche Baukonstruktionen ausreichend gute Ergebnisse, wobei der relativ geringe Aufwand mit der kleinen Eintrittswahrscheinlichkeit eines Erdbebens und dem damit verbundenen geringen Schadensrisiko in einem vernünftigen Verhältnis stehen. Aussagen iiber mögliche Relativbewegungen zwischen Gebaude und Turbinenfundament sowie Beanspruchungen am Turbosatz und anderen Komponenten bekommt man mlt diesen quasistatischen Methoden nicht. Dafür sind dynamische Rechenverfahren erforderlich, wie sie für sicherheitstechnisch wichtige Anlagenteile eines Kernkraftwerkes angewandt werden.

\subsection{Dynamische Methoden}

\section{Die drei in Frage kommenden Methoden}

- direkte numerische Integration

- Zeitablaufmethode (time history method) mit der Modalen Analyse

- Antwortspektrummethode (response spectrum method)

sind in der Literatur hinrelchend beschrieben und werden hier als bekannt vorausgesetzt. (Siehe z.B. [3] )

Im folgenden wird erläutert, wie die letztgenannte Methode, die in der Praxis allgemein üblich ist, für ein spezlelles Projekt angewendet wurde.

\subsubsection{Beschreibung einer speziellen dynamischen Berechnung}

Wie aus der Abbildung 3 zu sehen ist, sind die Gebäudeteile unter Berijcksichtigung der Blege- und Schubstelfigkeit als Stabmodelle aus finiten Elementen abgebildet. Die Massen der Deckenplatten und die ständigen Lasten sowle ein gewisser Teil der Verkehrslasten sind auf die Knotenpunkte in Höhe der Decken konzentrlert. Die dazwischenliegenden Wand- und Stitzentelle sind als gleichmäßig verteilte Massen in den Stäben berucks1chtigt. 
Die Fundamentplatte wird als starr angenommen. Die Elgenschaften des Baugrundes werden durch gedämpfte Translations- und Rotationsfedern und durch mitschwingende Bodenmassen simuliert, die Im Schwerpunkt der Fundamentplette angeordnet sind. Die Feder- und Dämpfungswerte hängen ab von den Bodeneigenschaften, der Gebäudemasse und den Massenträgheitsmomenten um die Achsen der Fundamentplatte.

D1. Turbinentischplatte wird als durchgehender Balken idealisiert und die Turbinengehäuse nur durch 1hre Massen im entsprechenden Abstand bericksicht1gt. Die durchgehende Turblnenwelle hat gegenuber der Tischplatte elne vernachlässigbar kleine stelfigkelt und ist zum anderen sehr oft gelagert, so daB sie für Erdbebenerregungen als starr angenommen werden kann. Ihre Masse wird deshalb anteilmäß1g den Gehäusemassen zugete1lt. Die Kondensatoren unter den Niederdrucktellen werden durch auskragende Massen bericksichtigt.

Die Federn werden in jeder Federebene mit thren entsprechenden Stelfigkelten an das elastische Gebäude- und Turbinenmodell gebunden.

Gebăude, Baugrund und Turbosatzfundament-Dämpfer haben unterschiedliche Dämpfung, die als Prozentsatz der kritischen Dämpfung gegeben ist. Fur die Berechnung wird jedoch die zu jeder Elgenfrequenz gehörige krit1sche Dämpfung fur das Gesamtsystem Gebäude-Baugrund-Turbinenfundament benötlgt. Diese wird aus der antelligen Verformungsenergie der elnzelnen Substrukturen mit unterschiedlichen Dämpfungen gew1chtet.

Relativverschiebungen, Relativgeschwindigkeiten und Absolutbeschleunigungen werden aus den zuvor ermittelten Elgenwerten und aus dem Beschleunlgungs-Antwortspektrum getrennt fir die Längs-, Quer- und Vertikalrichtung nach der Response Sepctrum Analysis berechnet. Es wird die Ub11che quadrat1sche Mittelwertblldung verwendet.

Aus den Absolutbeschleunigungen erhält man durch Multılikation mit den zugehörlgen Massen die infolge des Erdbebens auftretenden Zusatzkräfte fur das Gebäude.

Die Relativverschlebungen dienen zur Festlegung von Fugenbreiten und zur Ermittlung der Kräfte in den Federelementen des Turbinenfundamentes.

Aus den Relativgeschwindigkeiten an den Auflagerpunkten des Turbosatzfundamentes werden die Kräfte in den Dämpfern berechnet. 
Feder- und Dämpfungskräfte sind um $90^{\circ}$ phasenverschoben. Die maximale Kraft (Feder + Dämpfer) erhält man somlt durch geometrische Addition.

\subsection{Folgerungen sus den dynamischen Berechnungen}

In Abhäng1gkelt von der angenommenen Erdbebenintensität (Betriebserdbeben) werden aufgrund der Berechnungen folgende konstruktive Ertüchtigungsmaßnahmen gegenüber einem Maschinenhaus ohne Erdbebenauslegung notwendig:

\subsubsection{Kleines Erdbeben}

Bis zu einer Erdbebenintens1tät von I 5 sind keine Ertuchtigungsmaßnahmen auf der Turbosatzselte und nur geringe Verstärkungen (höhere Bewehrung) auf der Gebäudeselte erforderlich.

\subsubsection{M1ttleres Erdbeben}

Be1 einem mittleren Erabbeben ( $I \leq 6)$ genugt die Gebäudeverstärkung durch höhere Bewehrung meist nlcht, sondern es werden auch einlge Änderungen des Gebäudekonzeptes durch Elnplanung zusätzlicher Ausstelfungselemente wie Ausbildung der Längs- und Glebelwände als Scheiben oder stärkere Stützen notwendig.

Der von den Federkörpern zusätzlich aufnehmbare Federweg liegt in vertikaler und horizontaler Richtung im Millimeterberelch. Bel einer Uberschreitung werden die Federkörper plastiflzlert und es besteht die Gefahr des Aneinanderschlagens von Turbosatzfundament und Gebäude (Hammering-Effekt). Wle dle Erfahrungen aus dem Erdbeben in Managua/ Nicaragua 1972 gezeigt haben, entstehen dadurch die größten Schäden am Turbosatz.

Um das zu verhindern, milssen Dämpfer vorgesehen werden. Elne Fotografle und eine typlsche Anordnung von Federkörper und Dämpfer sind in den Blldern 4 und 5 wiedergegeben.

Auf der Turbosatzse1te 1st elne Verstärkung der Fixpunkte, der Gehäusefuhrungen und Distanzschrauben notwendig, um elne ausrelchende Verbindung zwischen den Gehäusen und dem Fundament herzustellen, und elne Ertüchtigung der Lager, um eine slchere Fihrung der Welle gewährleisten zu können. Darüberhinaus ist dle Frage elnes Bruches einer Steuerflussigke1tsleitung zu liberprifen. Die Leitungen werden gegen hydraulische Druckstöße ausgelegt, wodurch sie auch gegen äußere Erschïtterungen resistent werden. 
Belm Bruch einer Steuerflüssigkeitsleitung bleibt der Folgeschaden gering, weil 1. des Schutzkonzept so aufgebaut 1st, daß bei zunehmendeII Steuerflussigkeitsdruck die Dampfvent1le automat1sch schließen und 2. die Flussigke1t schwer entflammbar 1st (BrandschutzmaBnahmen).

\subsubsection{Starkes Erdbeben}

Für elne Intensität I> 6 ist es notwendig, eln aseism1sches Gebäudekonzept unter Bericks1chtigung ein1ger der folgenden Ertüchtigungsmaßnahmen zu entwerfen. Dazu gehören:

- Verstärkung und Verbreiterung der Grundplatte

- tiefere Grindung des Gebäudes

- möglichst gleichbleibende Grindungstiefe

- Wegfall der Maschinenhaushalle bzw. Ersatz durch eine lelchte Stahlkonstruktion. Wenn dies aus Schallschutz- oder Witterungsgridnden nicht möglich 1st, Vergrößerung der Abmessungen der AuBenstïtzen und Wahl einer leichten Dachkonstruktion.

- Vertellung der Aussteifungselemente und Massen In der Weise, daß Schubmittelpunkt und Massenschwerpunkt der Gebäudequerschnitte zusammenfallen und die Anregung von Torsionsschw1ngungen vermieden wird. In den meisten Fällen bedeutet das den Verzicht auf die bautechnisch gúnstige Dehnungsfuge in Gebäudem1tte, die zur Reduzlerung der Schwind- und Temperaturbelastungen sowie zum Ausgleich von Setzungsunterschieden üblicherweise vorgesehen wird.

- Reduzierung der Kranbahnweiten auf das montagetechnisch erforderliche Mindestmaß.

Ein typlsches Beisplel fur ein aseismisches Gebäudekonzept 1st im B1ld 6 wiedergegeben. Die Unterschlede gegenüber dem nicht ertüchtigten Konzept im Bild 1 sind deutlich zu erkennen.

Für das Turbosatzfundament ist die kritische Stelle der Querriegel, an dem über das Achsiallager dle gesamten erdbebeninduzlerten Längskräfte aus dem Wellenstrang des Turbosatzes über Biegung und Torsion eingele1tet werden. Die Verbreiterung des Querrlegels hat elne weitgehende Umkonstruktion der Turblnenwelle zur Folge. W1ll man das vermeiden, so bleibt der Ausweg uber die Ausstelfung des Rlegels durch elne Fachwerkkonstruktion. Gegenlber dem mittleren Erdbeben ist eine gröBere Anzahl von Dämpfern erforderlich. 
Auf der Turbosatzseite sind die Ertüchtigungsmaßnahmen analog denen des mittleren Erdbebens.

Die Gle1tlagerflächen bleiben bel der kurzen Uberbeanspruchung Intakt, well eine unzulässige Erwärmung erst bei längerer Uberbeanspruchung auftritt.

\section{Parameteruntersuchung}

Um standort- und gebäudeunabhäng1ge Aussagen machen zu können, wurden Baugrundparameter und Gebäudekonzeptionen varilert. Als Belastung wurde das für den Standort Goesgen/Schwelz festgelegte Betrlebserdbeben angenommen.

\subsection{Varlation der Baugrundparameter}

Untersucht wurden die Fälle weicher Baugrund, mittelfester Baugrund und fester Baugrund (starre Einspannung). Die Ergebnisse sind in den Bildern 7 bis 9 fir die 3 Hauptrichtungen dargestellt, wobei die Beschleunigung des Turbosatzes der Mittelwert aus den Teilbeschleunlgungen von Turbosatz und Kondensator ist. Für das Gebäude 1st der maximale Beschleunigung swert gewählt worden.

Die Kurven zeigen elnmal den typischen Verlauf des Abfalls der Gebäudebeschleunlgung m1t abnehmender Bodensteifigkeit. Zum anderen stelgt die Turbosatzbeschleunlgung m1t fallender Gebäudebeschleunigung an, was auf einen schwingungstilgungseffekt zurückzufübren ist.

\subsection{Variation der Gebäudekonstruktion}

Es wurde hierbei das Schwingungsmodell des Gebäudes so varliert, dab neben einem offenen Gebäude (zwe1 gegeneinanderstehende offene U-Proflle) der Effekt durch Verbindung beider Gebäudeteile mit einer durchgehenden Dachscheibe und das Verhalten eines geschlossenen Kastenprofils erfabt werden konnte. Der Baugrund wurde als mittelfest angenommen.

Die Ergebnisse sind auf den Blldern 10 und 11 für beide horizontale Richtungen aufgetragen. Auf die vertikale Rlchtung konnte verzichtet werden, well sich das Verhalten in verikaler Richtung, wie zu erwarten, dadurch nicht verändert hat.

Die Ergebnisse zelgen, daB mit zunehmender "Gebäudesteifigkeit" die Beanspruchungen Im Gebäude und an der Turbine abnehmen. Bei höheren Erdbebenbeanspruchungen ist es daher wirtschaftlich, auf die Anordnung von Dehnungsfugen Im Gebäude zu verzichten und die durch den Wegfall erforder- 
Ilche höhere Bewehrung für Temperatur- und Schwindbelastungen in Kauf zu nehmen.

\section{Zusammenfassung}

Im vorllegenden Bericht wurde erläutert, wle Maschinenhäuser von Kernkraftwerken mit Turbosätzen der Kraftwerk UnIon Baulinie erdbebensicher ausgelegt werden können, ohne daB das Betrlebsverhalten des Turbosatzes unglinstig beelnflußt wird. Es wurden die Ertuchtigungsmaßnahmen fur verschledene Erdbebenintensitäten diskutiert und ein1ge Ergebnisse elner Parameterstudie angegeben.

\section{L1teraturverzelchnis:}

[1] PROBST, P.H., JOYCE, J.S.,

"The Development of Helical-Spring

Foundations for Large

Steam Turbine - Generators"

American Power Conference,

Chlcago, Illinois, Apr1l 19, 1972

[2] Societe de diffusion des

technlques du bâtıment et des

travaux publics, Paris.

RGgles Parasismiques 1969 et

Annexes, October 1970.

[3] WIEGEL, R.L., "Earthquake Engineering", Prentice-Hall, Inc., Englewood Cliffs, N.J. 


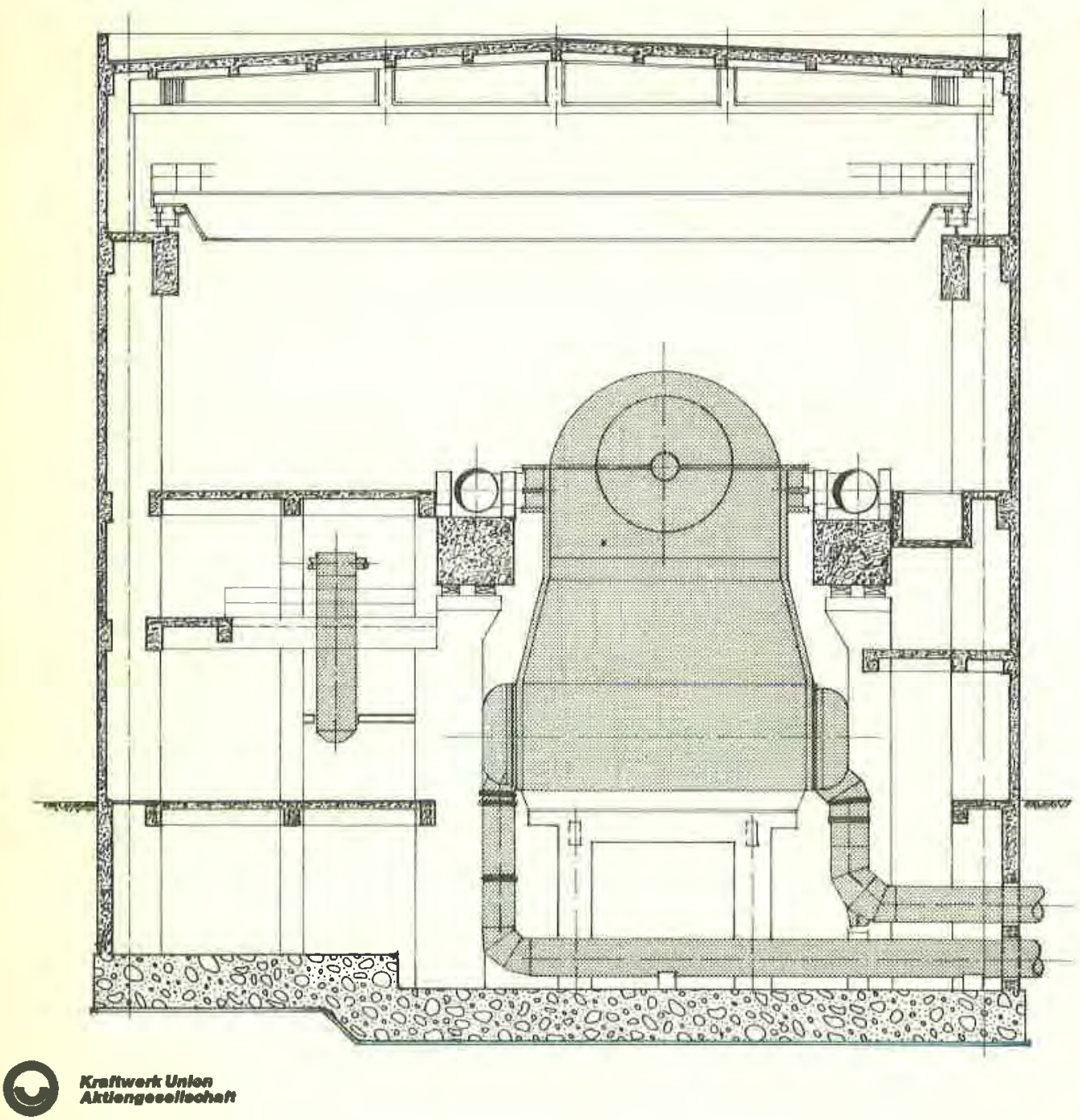

Bild 1 : Querschnitt durch ein Maschinenhaus 

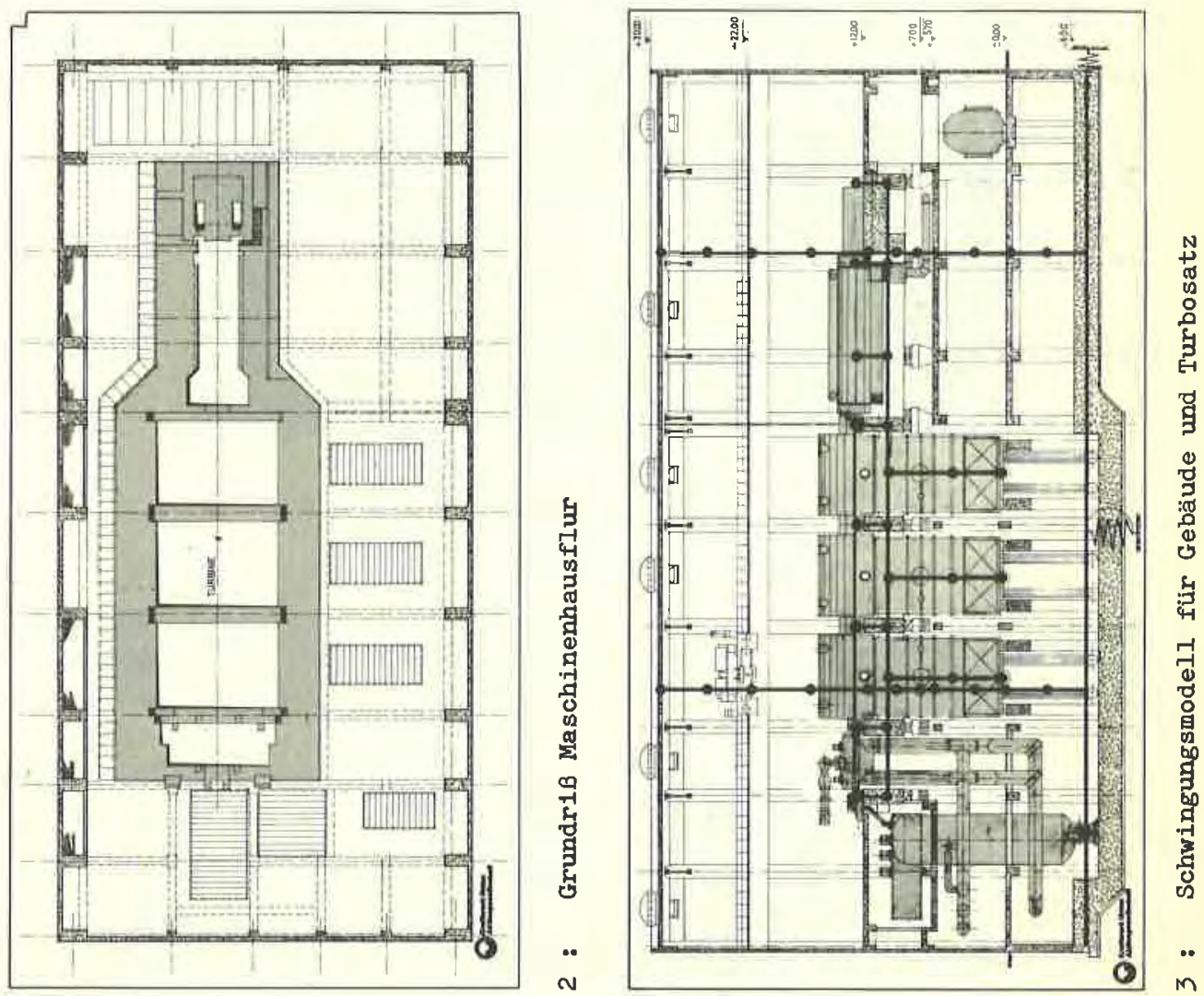


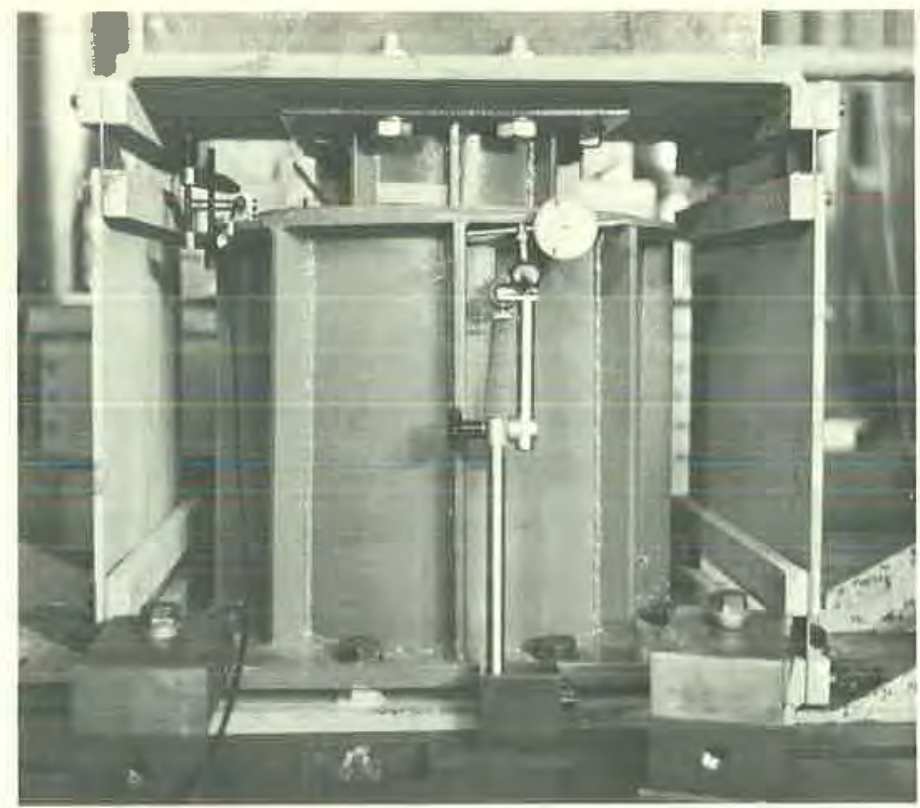

4: Dämpfer in elner Versuchseinrichtung zur Ermittlung der Dämpfereigenschaften

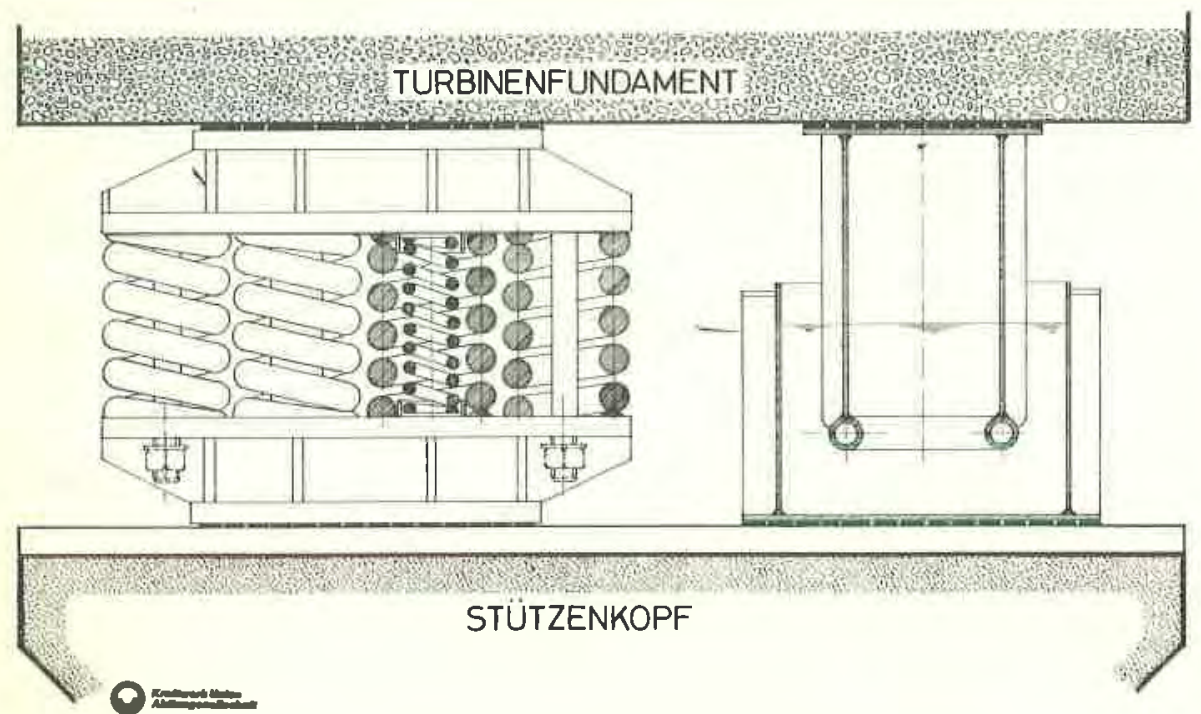

5 : Typ1sche Anordnung eines Federkörpers und Dämpfers 


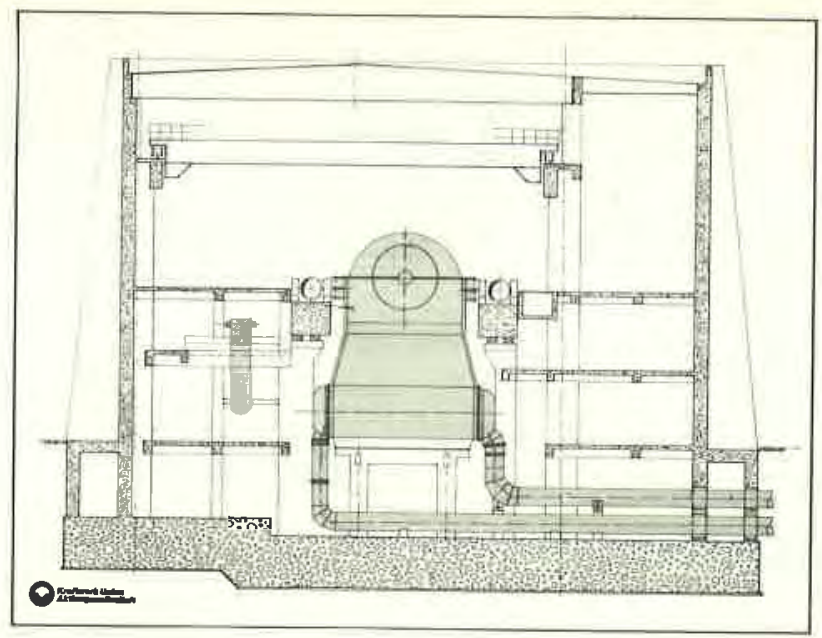

6 : Ase1smisches Gebäudekonzept

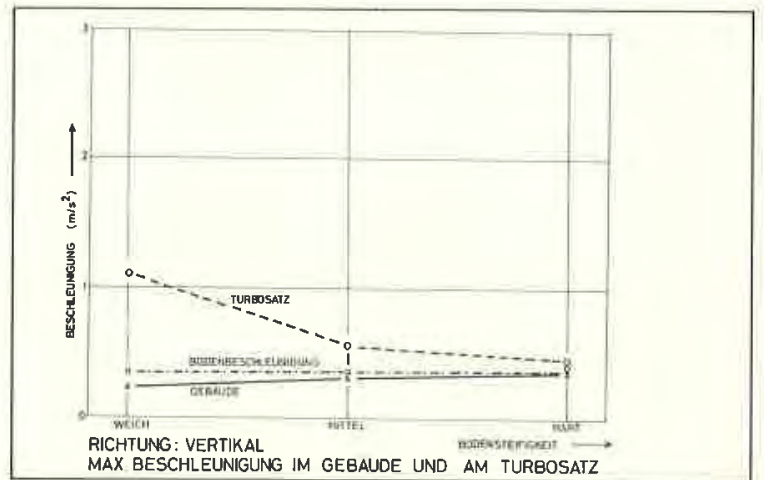

7 : Variation der Bodenparameter, vertikal

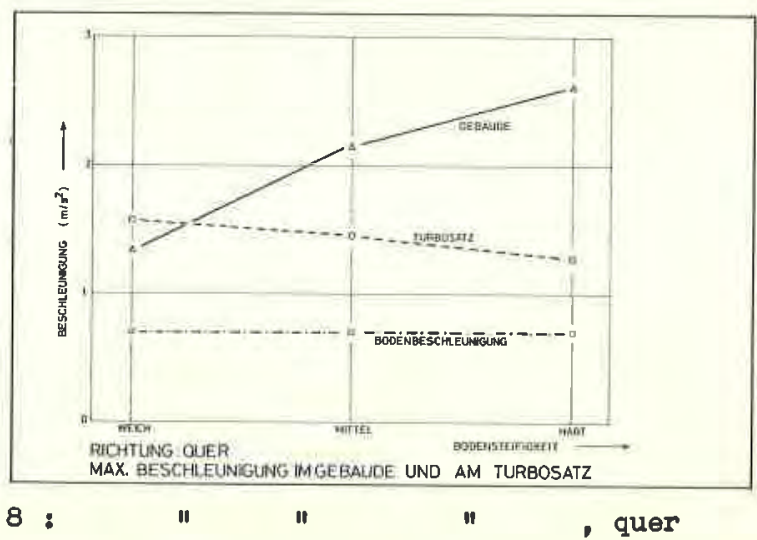




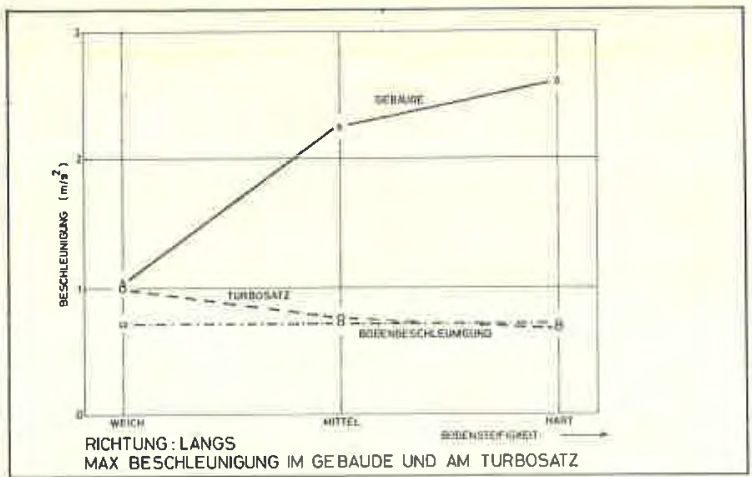

9 :

"

II

n

, längs

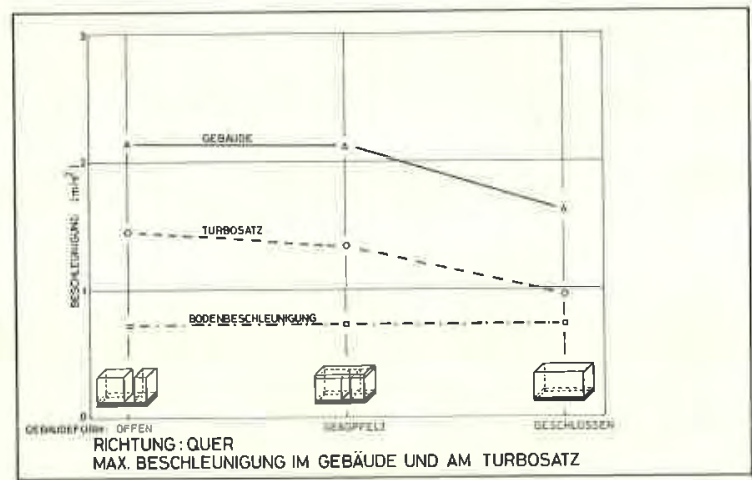

10 : Variatıon der Gebäudekonstruktion, quer

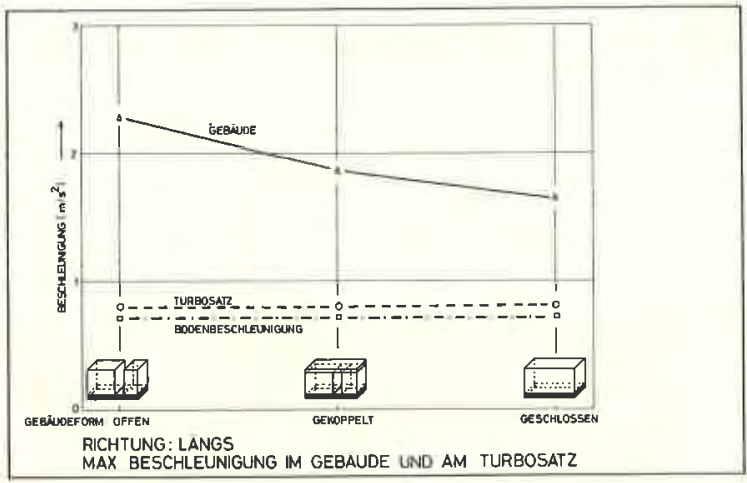

$11:$

"I

"

n

, längs 